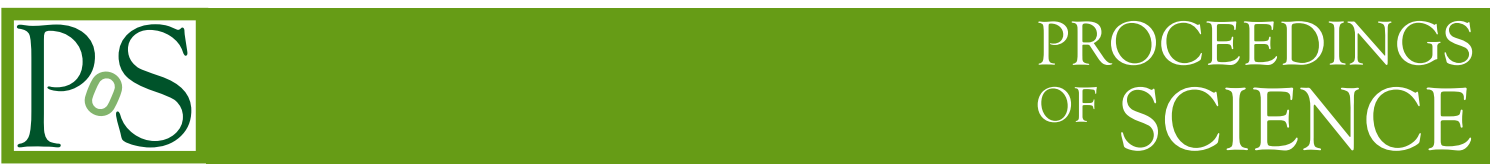

\title{
Progress on Excited Hadrons in Lattice QCD
}

\author{
John Bulava* \\ CERN, Physics Department, 1211 Geneva 23, Switzerland \\ E-mail: john.bulava@cern.ch
}

The study of excited hadron spectra using Lattice QCD is currently evolving. An important step toward obtaining resonance parameters from Lattice QCD is the calculation of finite volume energy spectra. Somewhat more rigorous studies of finite volume spectra are currently possible and should be completed in the near future. The inclusion of disconnected diagrams is increasingly commonplace and the simplest systems which involve mixing between single- and multi-hadron interpolating fields are being studied. Advances in all-to-all algorithms which have been crucial in this progress are reviewed and a survey of current results is given. Nevertheless, such results are preliminary and a thorough discussion of systematic errors is required. We discuss several such sources of error, focussing on excited state contamination and the use of the generalized eigenvalue problem. Also, the calculation of matrix elements between finite volume Hamiltonian eigenstates is discussed.

CERN-PH-TH/2011-300

The XXIX International Symposium on Lattice Field Theory - Lattice 2011

July 10-16, 2011

Squaw Valley, Lake Tahoe, California

* Speaker. 
Excited hadrons are interesting to study on the lattice, as their properties cannot be calculated using perturbation theory and exhibit many features of strongly coupled QCD. Furthermore, there are several unresolved questions related to excited hadrons. For example, most quark model calculations of baryon spectra predict considerably more states than have been observed experimentally. This may be either because the quark models possess unphysical extra degrees of freedom or because most experimental analyses have focused on states which decay to $N \pi$. A summary of the current status of this 'missing resonance' problem can be found in Ref. [1].

In addition to missing resonances, it remains a puzzle why the quantum numbers of known hadrons can be predicted from the constituent quark model, which assumes that mesons contain two and baryons three quarks. States which have quantum numbers which do not fit these predictions are said to be 'exotic' and are the subject of experiments at JLab, Cleo-c, BES III, and PANDA (For an overview see Ref. [2]).

Lattice QCD calculations are necessarily performed at finite lattice spacing (a) and finite system size $(L)$. Extracting infinite volume resonance parameters from this finite volume data is difficult. The spectrum of the QCD Hamiltonian in infinite volume consists of stable single hadron states and a continuum of multi-hadron states above threshold. The situation in finite volume is quite different; resonance states which were unstable in infinite volume are now eigenstates of the finite volume Hamiltonian. Near threshold the finite volume energy eigenvalues are distorted from their non-interacting behavior as the spatial extent of the system is varied and an 'avoided level crossing' occurs. An analogous situation occurs in the study of string breaking in QCD [3], where an avoided level crossing occurs as the length of the string is varied.

Somewhat more formally, resonances are observed by experiment as rapid variations in the cross section as the center-of-mass energy moves through the resonance mass. This can be interpreted as a singularity in the $S$-matrix, which (by the LSZ reduction formula) corresponds to a singularity in the $n$-point correlation functions of the underlying quantum field theory. The singularity structure of a two-point correlation function with suitable quantum numbers is made apparent by employing the Källen-Lehmann representation:

$$
\Delta(p)=\int_{0}^{\infty} d \mu^{2} \rho\left(\mu^{2}\right) \frac{1}{p^{2}-\mu^{2}+i \varepsilon}
$$

where $\Delta(p)$ is the two-point function (the spinless case is shown here) in momentum space, and $\rho\left(\mu^{2}\right)$ is the spectral density. In infinite volume $\rho\left(\mu^{2}\right)$ consists of $\delta$-functions corresponding to stable particles below threshold and a continuum of states above threshold.

Conversely, in finite volume $\rho\left(\mu^{2}\right)$ consists entirely of $\delta$-functions corresponding to stable states. However, infinite volume scattering phase shifts may be extracted from finite volume lattice data below inelastic thresholds. This method [4] relates infinite volume elastic scattering phase shifts to the distortions of finite volume energy spectrum near thresholds mentioned above.

While elastic scattering phase shifts may be extracted in this manner, systematically extracting infinite volume resonance parameters from finite volume data is still an active area of research $[5,6$, 7]. Regardless, an important first step is to extract the spectrum of QCD in a finite box. This is hard enough, as there are many sources of systematic error which much be controlled. As resonances are expected to be spatially extended objects, large volumes are needed. Furthermore, extrapolations to the physical quark masses are complicated by the inapplicability of chiral effective theory at scales 

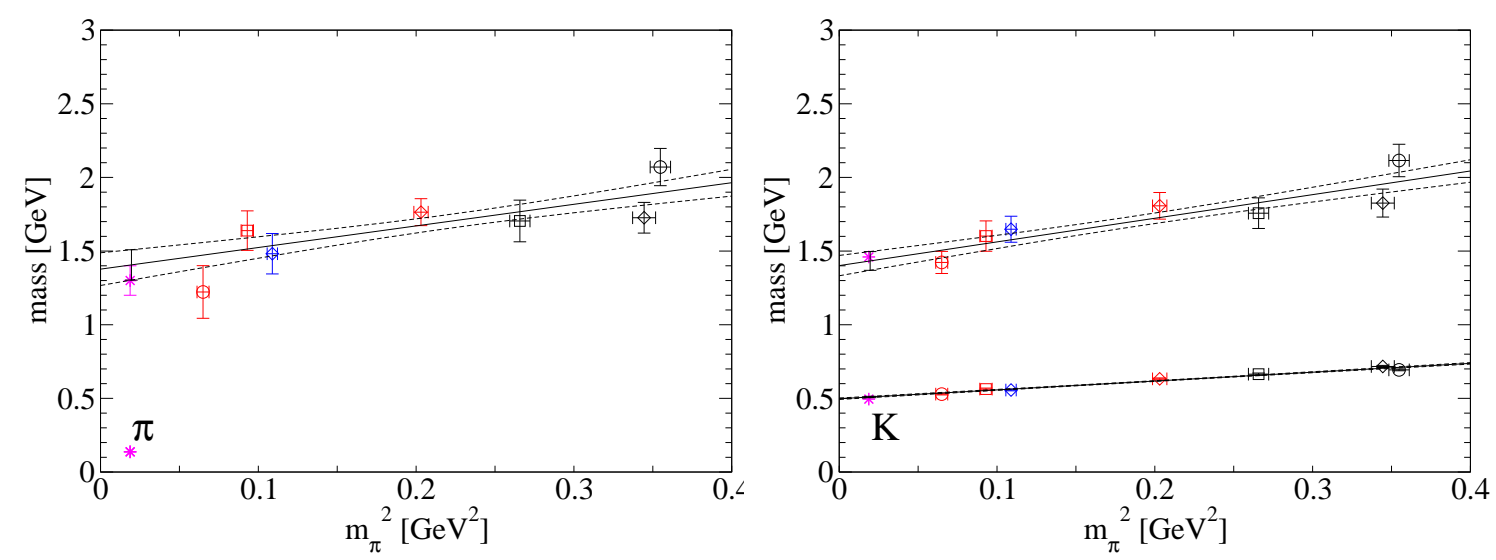

Figure 1: Preliminary results for the chiral behavior of some excited meson states [11]. Results for the pion channel are shown on the left, while results for the kaon channel are shown on the right. These spectra are calculated on ensembles with $a_{s}=a_{t}=0.13 \mathrm{fm}$ and $L_{s} \sim 2.2 \mathrm{fm}$, where $a_{s}$ and $a_{t}$ are the spatial and temporal lattice spacings (respectively) and $L_{S}$ is the spatial lattice extent. Multi-hadron operators are not included in analysis, which may result in systematic errors due to threshold effects.

around and above $m_{\rho}$. Additionally, higher energy resonances may suffer from large discretization effects so the continuum limit must be taken. Finally, in order to extract the energy of an excited state, a large basis of interpolating fields must be employed, and contamination from unwanted excited states is present.

Progress has been made recently toward determining the pion mass dependence of some low lying hadron resonances. Current Lattice QCD simulations are performed at unphysically large light quark masses and physical results must be obtained by extrapolating to the correct values. As discussed above, the use of chiral effective theory to predict the dependence of excited state energies on the quark mass is limited. Indeed, if thresholds open up as the pion mass is decreased toward the physical point, the chiral behavior of excited states may not even be analytic. Therefore, this dependence is largely unexplored and must be determined empirically. Preliminary calculations of the chiral behavior of some excited meson states are shown in Fig. 1, while examples for baryons are shown in Fig. 2. Additionally, a preliminary scan of excited baryon spectra at several pion masses can be found in Refs. [8,9] while preliminary results for $D$ mesons and charmionium states (without disconnected diagrams) can be found in Ref. [10]. However, all of these examples do not include multi-hadron operators in their analyses, which may introduce threshold-related systematic errors.

We now discuss in more detail the calculation of excited state energies in a finite volume. Energies in lattice field theory are typically extracted from the exponential fall-off of temporal correlation functions $C(t)=\langle\mathscr{O}(t) \overline{\mathscr{O}}(0)\rangle$ between interpolating fields $\mathscr{O}$ with particular quantum numbers. The energy of the lightest state with these quantum numbers is obtained from the asymptotic (in euclidean time $t$ ) single-exponential behavior of the correlation function. Excited states, however, can be extracted by forming a correlation matrix $C_{i j}(t)=\left\langle\mathscr{O}_{i}(t) \overline{\mathscr{O}}_{j}(0)\right\rangle$ between a set of 

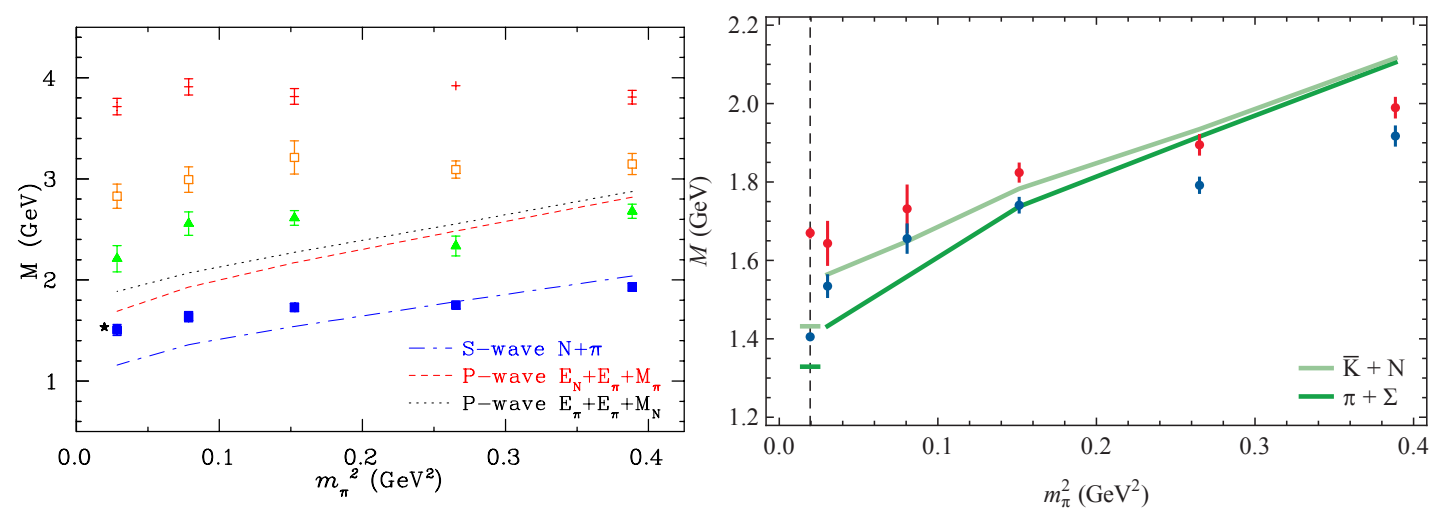

Figure 2: Preliminary results for the chiral behaviour of some excited baryon states. Results for the even parity nucleon channel are shown on the left [12] while states in the $\Lambda$ channel [13] are shown on the right. Results were obtained on ensembles with $a_{s}=a_{t}=0.09 \mathrm{fm}$ and $L_{s}=2.9 \mathrm{fm}$. On both plots, multi-hadron thresholds are indicated. For the $\Lambda$ channel, a partially quenched strange quark was used to reproduce the physical kaon mass. No multi-hadron operators were included in the analysis, which may result in systematic errors due to threshold effects.

interpolators $\left\{\mathscr{O}_{i}\right\}$ and solving the generalized eigenvalue problem (GEVP) $[14,4,15]$

$$
\begin{aligned}
C(t) v_{n}\left(t, t_{0}\right) & =\lambda_{n}\left(t, t_{0}\right) C\left(t_{0}\right) v_{n}\left(t, t_{0}\right) \\
\lim _{t \rightarrow \infty} E_{n}^{e f f}\left(t, t_{0}\right) & =\lim _{t \rightarrow \infty}-\partial_{t} \ln \lambda_{n}\left(t, t_{0}\right)=E_{n},
\end{aligned}
$$

where $E_{n}$ is the energy of the $n$th state interpolated by the $\left\{\mathscr{O}_{i}\right\}$.

The asymptotic corrections to $E_{n}^{e f f}\left(t, t_{0}\right)$ can take different forms based on the relation of $t_{0}$ to $t$. Generically, it has been proven [4] that asymptotically $E_{n}^{e f f}\left(t, t_{0}\right)=E_{n}+\mathscr{O}\left(\mathrm{e}^{-\left(E_{n+1}-E_{n}\right) t}\right)$, where $E_{n+1}$ is the energy of the state above $E_{n}$. This correction may be large in systems with closely spaced energy levels. However, if the condition $t_{0} \geq t / 2$ is maintained, it has been proven [15] that $E_{n}^{e f f}\left(t, t_{0}\right)=E_{n}+\mathscr{O}\left(\mathrm{e}^{-\left(E_{N+1}-E_{n}\right) t}\right)$, where $N$ is the dimension of the GEVP. In this manner, the corrections can be systematically improved by increasing the size of the GEVP basis. However, this may increase the condition number of the resultant correlation matrix, resulting in larger statistical errors on the GEVP eigenpairs.

Due to these two considerations, selecting a GEVP basis is a delicate procedure. As mentioned above, increasing the number of operators in the basis may decrease the asymptotic corrections but care must be taken to prevent a large condition number. A simple procedure to construct a basis of operators amounts to applying different levels of Gaussian smearing. However, in the case of pseudoscalar static-light mesons, it has been suggested [16] that interpolating operators constructed from different smearings may have poor overlap with excited states. While ideal for extracting ground state properties, other types of interpolators may be more suitable for excited states. Spatially extended operators designed to transform irreducibly under lattice symmetries [17, $18,19]$ have been effective in extracting higher excited states.

The GEVP may also be used to define 'optimized' interpolating fields [14] $\mathscr{O}_{n}^{\text {opt }}=\left(v_{n}\right)^{i}\left(t, t_{0}\right) \mathscr{O}_{i}$, where $t$ and $t_{0}$ are typically fixed. Correlation functions of these optimized fields are the diagonal elements of the rotated correlation matrix and have increased overlap with the $n$th state. However, 

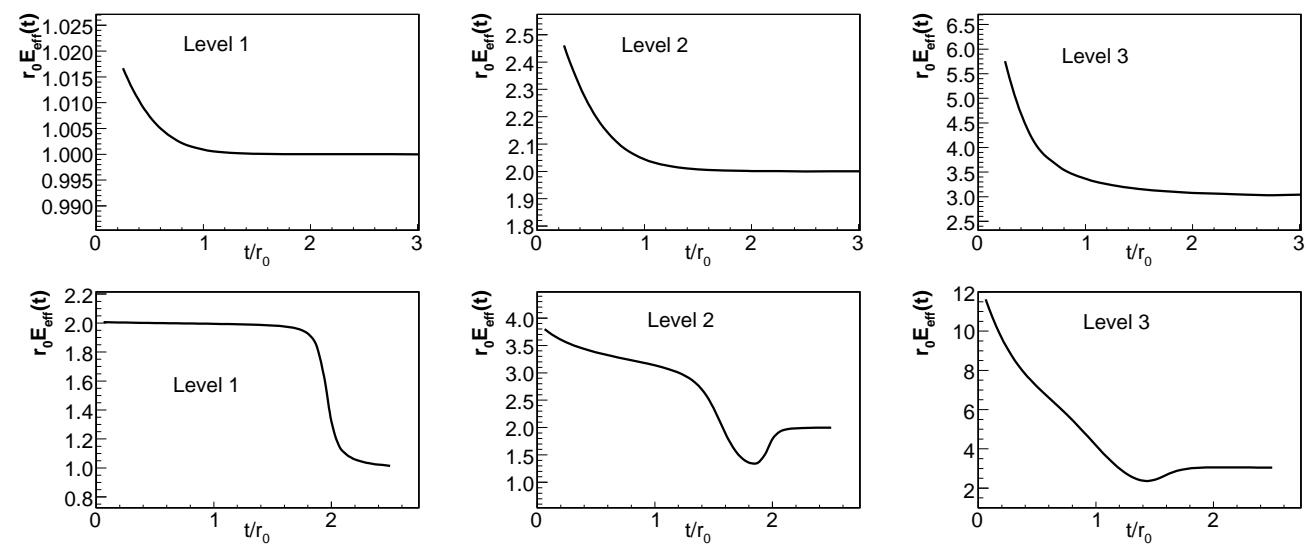

Figure 3: Results from a $3 \times 3$ GEVP in a toy model consisting of 20 evenly spaced energy levels. Top row: The effective energies for the first three levels when the matrix of overlaps $\left(\psi_{i m}\right)$ is given canonical values. Bottom row: The effective energies when the overlaps of all operators with the first level $\left(\psi_{i 1}, i=1,2,3\right)$ are decreased by an order of magnitude. In the case where a low-lying state has small overlaps with all operators in the basis, the asymptotic behaviour does not set in until large times. In realistic computations statistical errors grow exponentially with time and such non-asymptotic behaviour could be mistaken as a 'false plateau'.

mixing can occur and the off-diagonal elements of the rotated correlation matrix must be small to extract excited state energies.

In practice, the maximum temporal separation at which the correlation functions can be evaluated is limited by the signal-to-noise problem (see e.g. Refs. [20,21]) and the finite temporal extent of the lattice. It is therefore crucial that $E_{n}^{e f f}\left(t, t_{0}\right)$ behaves asymptotically as quickly as possible. Although the asymptotic corrections to effective energies in the GEVP are independent of which interpolators are included in the set, the time at which asymptotic behavior sets in can vary. In particular, if there is a state below $E_{n}$ with which the operators have little overlap, the asymptotic behavior may set in only at large times.

To illustrate this point we consider a toy model, which is solved numerically (variants of which have been considered in Ref. [16]). The model is specified by providing an analytic expression for the correlation matrix

$$
C_{i j}^{2 p t}(t)=\sum_{m} \psi_{i m} \psi_{j m}^{*} \mathrm{e}^{-E_{m} t}
$$

where $r_{0} E_{m}=m, m=1 \ldots 20$ and the $3 \times 20$ matrix $\psi$ is chosen empirically from an approximate calculation of the overlaps in the psuedoscalar static-light meson system [16]. The results from the $3 \times 3$ GEVP are shown in Fig. 3. The effective energies corresponding to 'normal' values of the $\psi$ matrix plateau rather quickly and have corrections of a standard form. When $\psi_{i 1}, i=1, . .3$ are decreased by two orders of magnitude the situation is quite different, however, and the asymptotic behavior sets in only at large times. While statistical errors are not included in this model, it seems plausible that in the second case with statistical errors one may mistake non-asymptotic behavior as a 'false plateau', illustrating the danger of states with small overlaps with all the operators in the basis. 

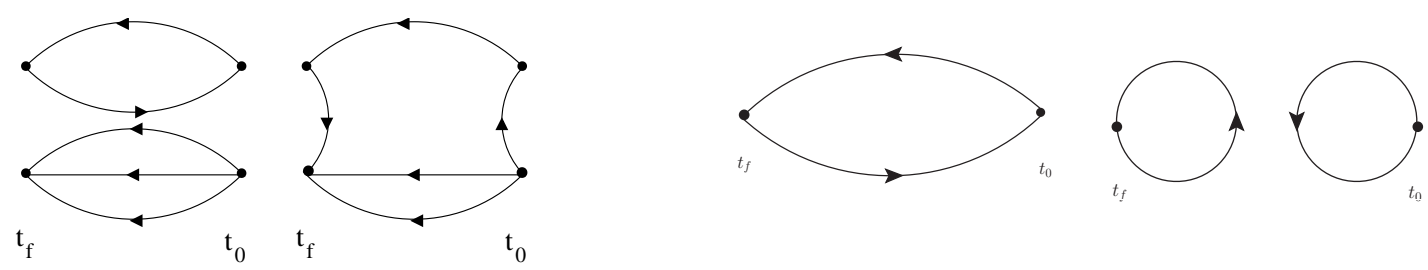

Figure 4: Types of valence quark line diagrams which must be included for a comprehensive scan of the finite volume excited hadron spectrum. Left: Valence quark line connected and disconnected diagrams contributing to a nucleon-pion correlation function. Right: Connected and disconnected diagrams contributing to a flavor singlet meson correlator.

Given these considerations, a reliable calculation of the hadron spectrum above threshold should include both single and multi-hadron interpolating operators (and perhaps other 'exotic' operators as well [22]). These multi-hadron operators require all-to-all propagators, which describe quark propagation from all initial sites to all final sites. This is to be contrasted with 'point-to-all' propagators, which describe quark propagation from a single initial site to all final sites. All-to-all propagators are also required in flavor-singlet single hadron states and are therefore necessary for a comprehensive scan of the meson spectrum.

The situation can be illustrated by examining valence quark line diagrams. Four such diagrams are shown in Fig. 4. When evaluating e.g. a nucleon-pion correlation function both valence quark line connected (where all valence quark lines propagate between the initial and final times) and valence quark line disconnected diagrams will contribute. Similarly, for flavor singlet mesons both connected and disconnected diagrams will contribute.

Quark propagators are obtained by inverting the lattice Dirac matrix. This matrix is of large $\left(12 \times\left(L_{s} / a_{s}\right)^{3} \times\left(L_{t} / a_{t}\right)\right)$ dimension and its inverse cannot be calculated directly. Instead, the action of the inverse on a 'source' vector can be obtained by the use of suitable algorithms. Clearly, it is not feasible to evaluate all-to-all propagators naively, i.e. by inverting on $\left(12 \times\left(L_{s} / a_{s}\right)^{3} \times\left(L_{t} / a_{t}\right)\right)$ sources, each with support on a single space-time point, spin, and color.

A novel alternative to naive all-to-all has recently been proposed [23]. This 'distillation' method calculates quark propagation from a subspace spanned by the low-lying eigenmodes of the gauge-covariant Laplace operator to all sites. The projection onto this subspace may be viewed as a 'smearing' procedure which creates interpolators with enhanced overlap with the low-lying states of interest. Indeed this projection operation preserves all the symmetries of the unsmeared propagator and the width of the smearing operator may be controlled by the number of low-lying modes contained in the subspace (see Fig. 5).

Distillation allows one to compute exact all-to-all propagation from the subspace spanned by the low-lying modes of the gauge-covariant Laplace operator and requires a number of Dirac matrix inversions proportional to the dimension of this subspace. Unfortunately, the number of modes required to maintain a constant smearing radius increases with the spatial volume. As the cost of each Dirac matrix inversion is also proportional to the volume, the total cost of this algorithm scales like $\sim L_{s}^{6}$. This can be seen by examining the density of the low-lying eigenmodes for several volumes, shown in Fig. 5.

Nevertheless, distillation has been useful in small spatial volumes $\left(L_{s} \lesssim 2.5 \mathrm{fm}\right)$. Results from 

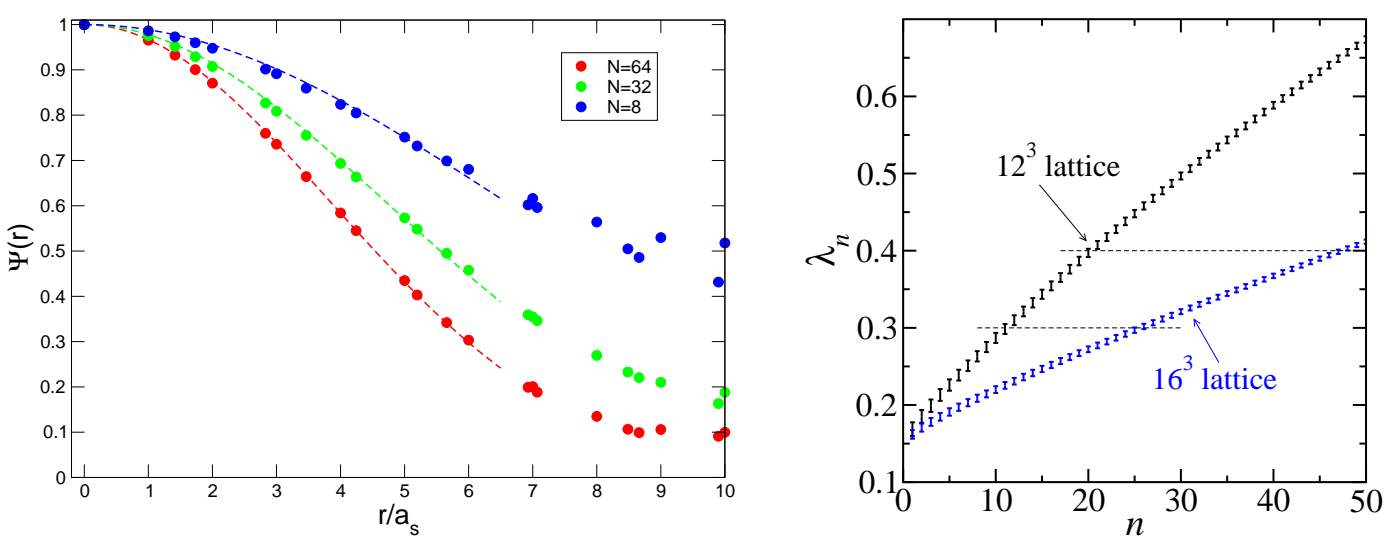

Figure 5: Left: Profile of the smearing operator as a function of the number of eigenvectors on an $n_{f}=2+1$ lattice with $a_{s}=3.5 a_{t}=0.12 \mathrm{fm}$ and $L_{s}=2.4 \mathrm{fm}$ (see Ref. [23]). Right: Low-lying eigenvalue spectra of the gauge covariant Laplace operator for two volumes, taken from Ref. [24]. Here $a_{s}=3.5 a_{t}=0.12 \mathrm{fm}$ and $L_{s}=1.4,1.9 \mathrm{fm}$. The number of modes contained between the dashed lines increases linearly with the spatial volume.
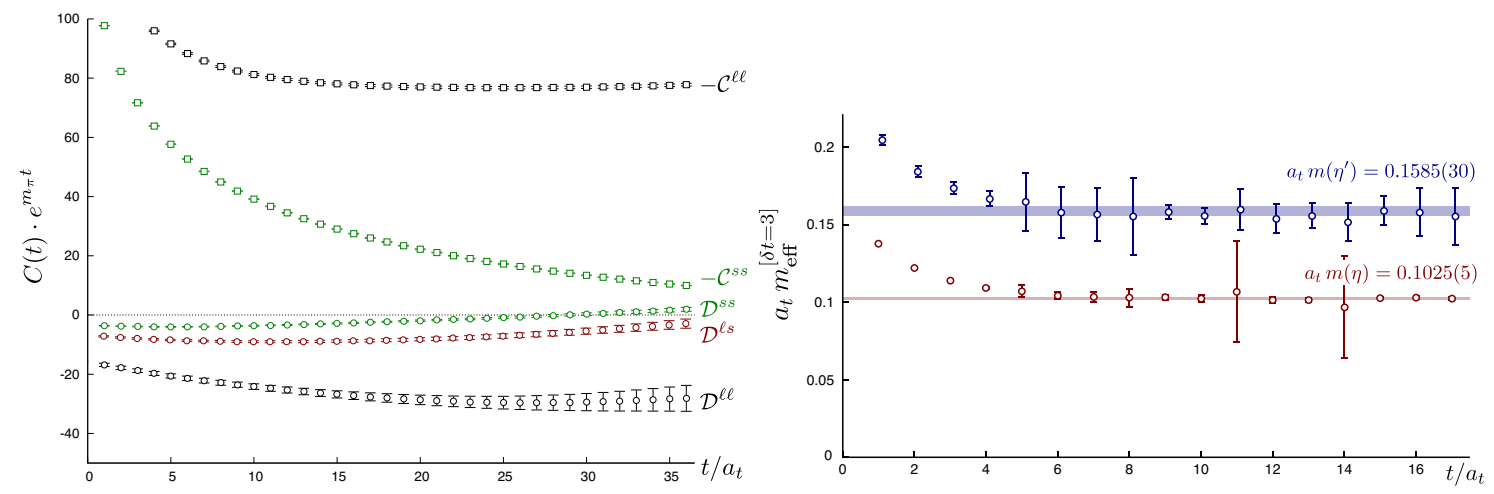

Figure 6: Results of a preliminary calculation of the isoscalar meson spectrum (Taken from Ref. [28]) on an $n_{f}=2+1$ lattice with $a_{s}=3.5 a_{t}=0.12 \mathrm{fm}, m_{\pi}=400 \mathrm{MeV}$, and $L_{s}=1.9 \mathrm{fm}$. Left: Valence quark connected and disconnected contributions to an isoscalar scalar correlation function. Disconnected contributions are labeled ' $D$ ', while connected contributions are labeled ' $C$ '. Right: the effective energy for the lowest states in the $\eta$ and $\eta^{\prime}$ meson channels. A $\delta t=3$ approximation of the temporal derivative is used.

a preliminary calculation of isoscalar meson spectra using distillation are shown in Fig. 6, while preliminary charmonium results which use distillation are shown in Fig. 7. Distillation has also been used in calculations of $\pi-\pi$ scattering phase shifts (see Fig. 11 and Refs. [25, 26]) as well as preliminary excited baryon [9] and isovector meson [27] spectrum calculations.

Clearly, in order to move to large volumes a new all-to-all algorithm must be devised. One promising candidate is the 'stochastic LapH'(Laplacian Heaviside) approach [24]. This introduces noise in the subspace spanned by the low-lying modes only. Of course this noise may be 'diluted' [30] in the spin, time, and eigenvector indices. The superiority of these types of diluted stochastic sources over conventional dilution is illustrated in Fig. 8.

Furthermore, the volume dependence of the stochastic LapH method is relatively mild for a moderate amount of dilution. Formally, this means that the number of dilution projectors (and thus 


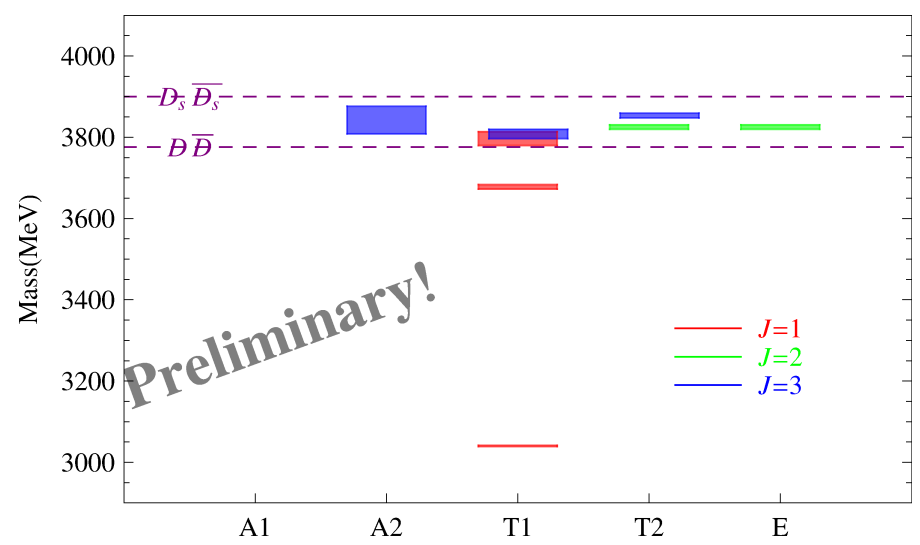

Figure 7: Preliminary results for charmonium spectra (taken from Ref. [29]) on an $n_{f}=2+1$ lattice with $a_{s}=3.5 a_{t}=0.12 \mathrm{fm}, m_{\pi}=400 \mathrm{MeV}$, and $L_{s}=1.9 \mathrm{fm}$. Multi-hadron operators were not included in the analysis and non-interacting multi-hadron energies are denoted by dotted lines.
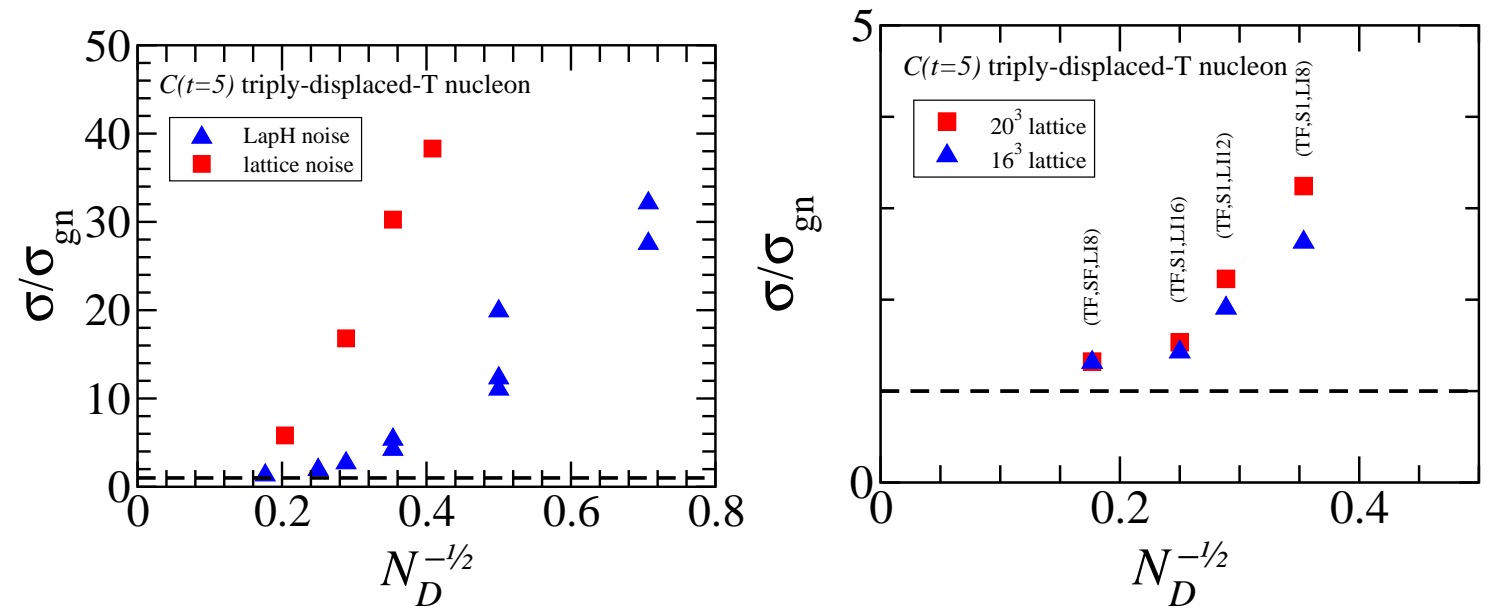

Figure 8: Properties of 'stochastic LapH' noise, taken from Ref. [24]. Left: The ratio of the error using stochastic LapH estimates to the error using the exact (distillation) method for a typical observable (triangles), plotted against $N_{D}^{-1 / 2}$, where $N_{D}$ is the number of dilution projectors. Conventional dilution (squares) is also shown for comparison. Results were obtained on a $n_{f}=2+1$ ensemble with $a_{s}=3.5 a_{t}=0.12 \mathrm{fm}$, $m_{\pi}=400 \mathrm{MeV}$, and $L_{s}=1.9 \mathrm{fm}$. Right: the same quantity for two volumes using stochastic LapH noise and dilution. Results were obtained on a $n_{f}=2+1$ ensemble with $a_{s}=3.5 a_{t}=0.12 \mathrm{fm}, m_{\pi}=400 \mathrm{MeV}$, and $L_{s}=1.9 \mathrm{fm}$ (triangles) and $2.4 \mathrm{fm}$ (squares).

the number of inversions) may be held constant as the volume is increased, without degrading the quality of the stochastic estimate. The cost now scales as $\sim L_{s}^{3}$ (due to the cost of each inversion) rather than the $\sim L_{s}^{6}$ required for exact distillation. This mild volume dependence is demonstrated in Fig. 8.

Of course, in our estimate of the cost scaling, we have considered the cost of the Dirac matrix inversions only. While at moderate lattice volumes $\left(L_{s} \sim 3 \mathrm{fm}\right)$ this dominates the computational cost, the calculation of the Laplacian eigenpairs must be taken into account. This is typically done using a variant of the Lanczos algorithm, with some form of polynomial preconditioning. In order to ensure numerical stability, a global reorthogonalization of the Lanczos vectors must be 

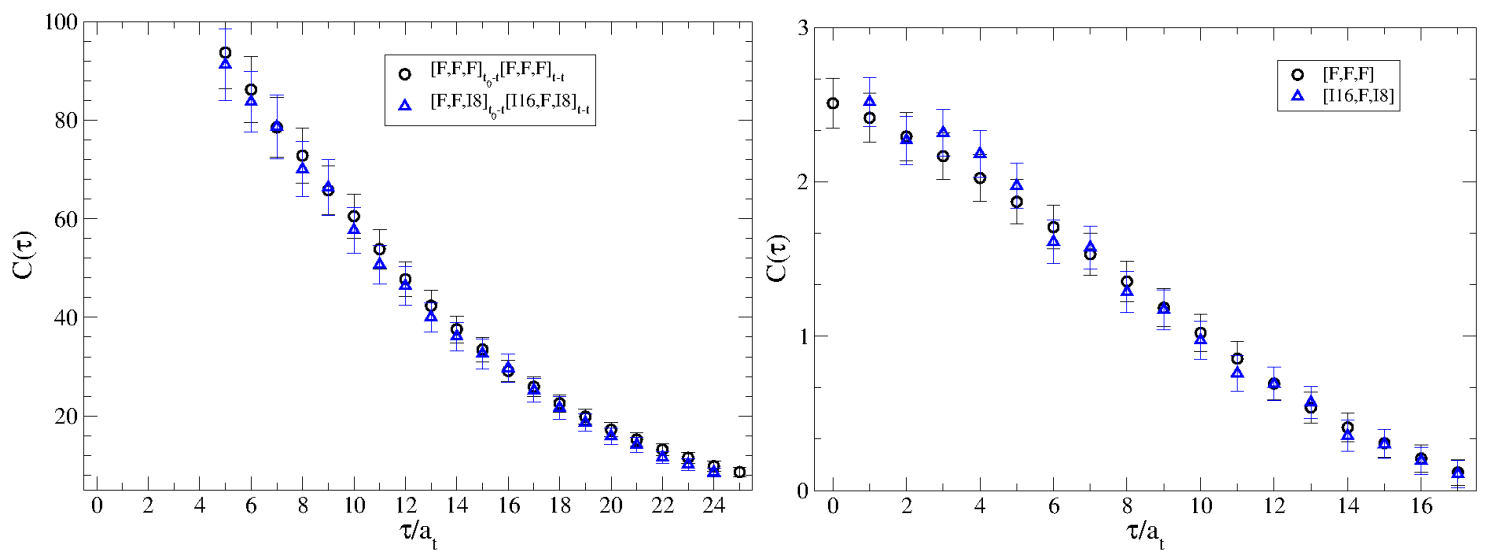

Figure 9: Comparison of stochastic LapH and distillation for disconnected diagrams (taken from Ref. [31]) on an $n_{f}=2+1$ lattice with $a_{s}=3.5 a_{t}=0.12 \mathrm{fm}, m_{\pi}=400 \mathrm{MeV}$, and $L_{s}=1.9 \mathrm{fm}$. Results using distillation are shown with black circles, while the ideal stochastic LapH dilution scheme is shown with with blue triangles. The left plot shows the 'box' diagram contribution to $I=0 \pi-\pi$ scattering while the right plot shows the disconnected contribution to a $I=0$ scalar correlation function. In the case of the scalar, the stochastic LapH dilution scheme requires 1024 inversions per configuration while the distillation result requires 16384 .

performed periodically. This reorthogonalization alone scales like $L_{s}^{9}$ so at larger volumes the cost of generating the Laplacian eigenpairs may become significant.

Apart from the valence quark line connected observable shown in Fig. 8, this method is also adequate for the estimation of disconnected diagrams. Flavor singlet correlation functions containing disconnected diagrams have a severe signal-to-noise problem, as the variance contains a component which is independent of the temporal separation. Therefore, even the variance of the exact all-to-all result is large, which allows one to reasonably estimate these diagrams using a moderate amount of dilution. Indeed, in Fig. 9 it is shown that an error similar to the exact distillation result can be obtained stochastically with e.g. a factor $\sim 16$ fewer Dirac matrix inversions per configuration.

After tests confirmed its utility, the stochastic LapH technique was applied to a preliminary calculation of the meson spectrum in a larger volume, the results of which are shown in Fig. 10. Although multi-hadron operators are not yet included, such a calculation would not have been feasible without the stochastic LapH approach.

Of course, there has been work on the inclusion of multi-hadron operators as well. The simplest system which exhibits mixing between single- and multi-hadron interpolating fields is the $I=1 \pi-\pi(\rho)$ sector. Several studies of the $\rho$-meson [33, 34, 35, 36] have been presented at this conference. A summary of the unquenched results as well as results from a smaller volume calculation of the $I=1 \pi-\pi$ phase shift using distillation are shown in Fig. 11.

Flavor singlet single hadron correlation functions also require valence quark line disconnected diagrams and can similarly be computed. Not only are flavor singlet meson spectra interesting in their own right, but may also appear in other channels as multi-hadron decay product states. To this end, flavor singlet interpolating operators must be studied both at rest and with non-zero spatial momentum if they are to be included as multi-hadron states in a GEVP analysis. Some 


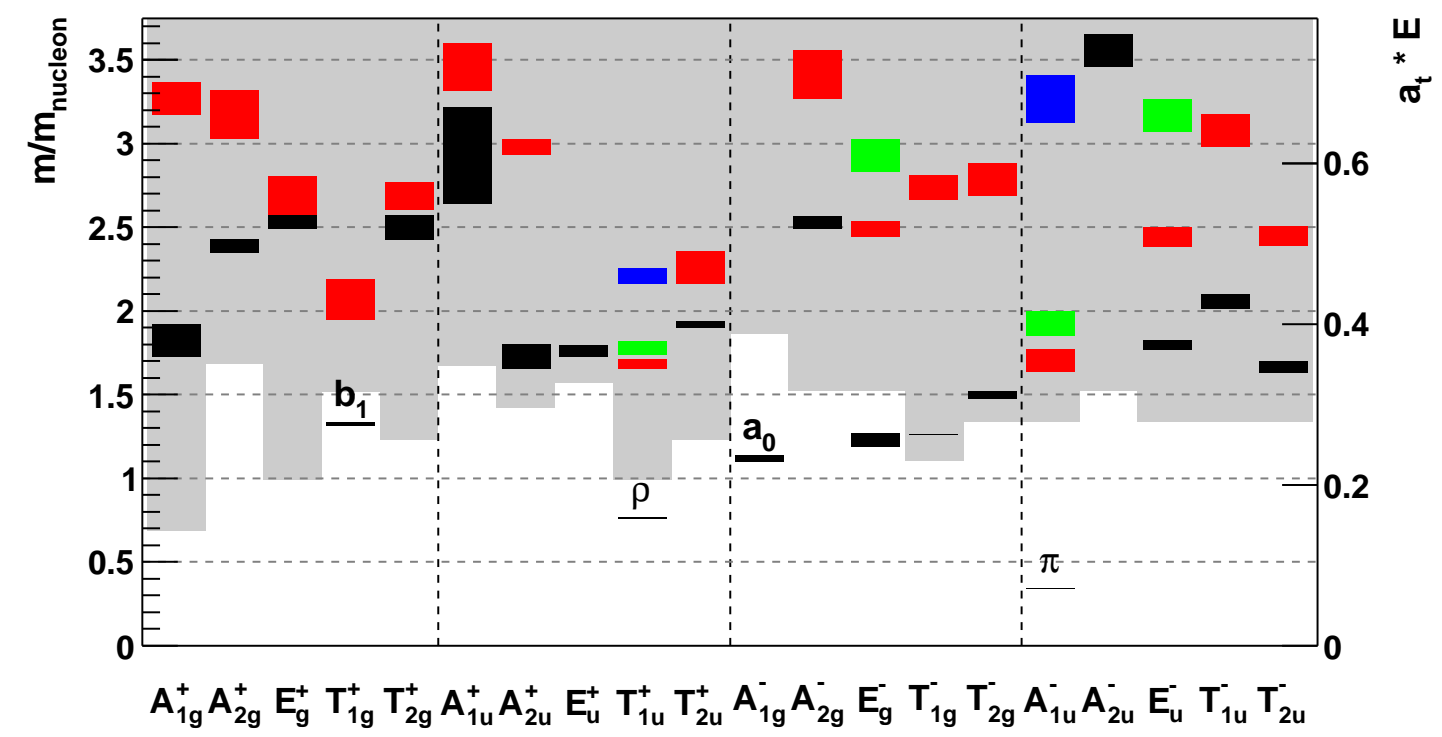

Figure 10: Isovector meson results (taken from Ref. [32]) on a $n_{f}=2+1$ ensemble with $a_{s}=3.5 a_{t}=$ $0.12 \mathrm{fm}, m_{\pi}=400 \mathrm{MeV}$, and $L_{s}=2.9 \mathrm{fm}$. The horizontal axis denotes different irreducible representations (irreps) of the lattice symmetry group. The subscript ' $\mathrm{g}$ ' denotes even parity irreps, while ' $\mathrm{u}$ ' denotes odd parity. The ' + ' and '-' labels refer to the G-parity of the irrep. $J=0$ states are found in the $A_{1}$ irrep, $J=1$ in the $T_{1}, J=2$ in the $E$ and $T_{2}$ irreps, and $J=3$ in the $A_{2}, T_{1}$, and $T_{2}$ irreps. Multi-hadron operators are not included in the GEVP analysis and multi-hadron thresholds for each set of quantum numbers are indicated by the shaded area.
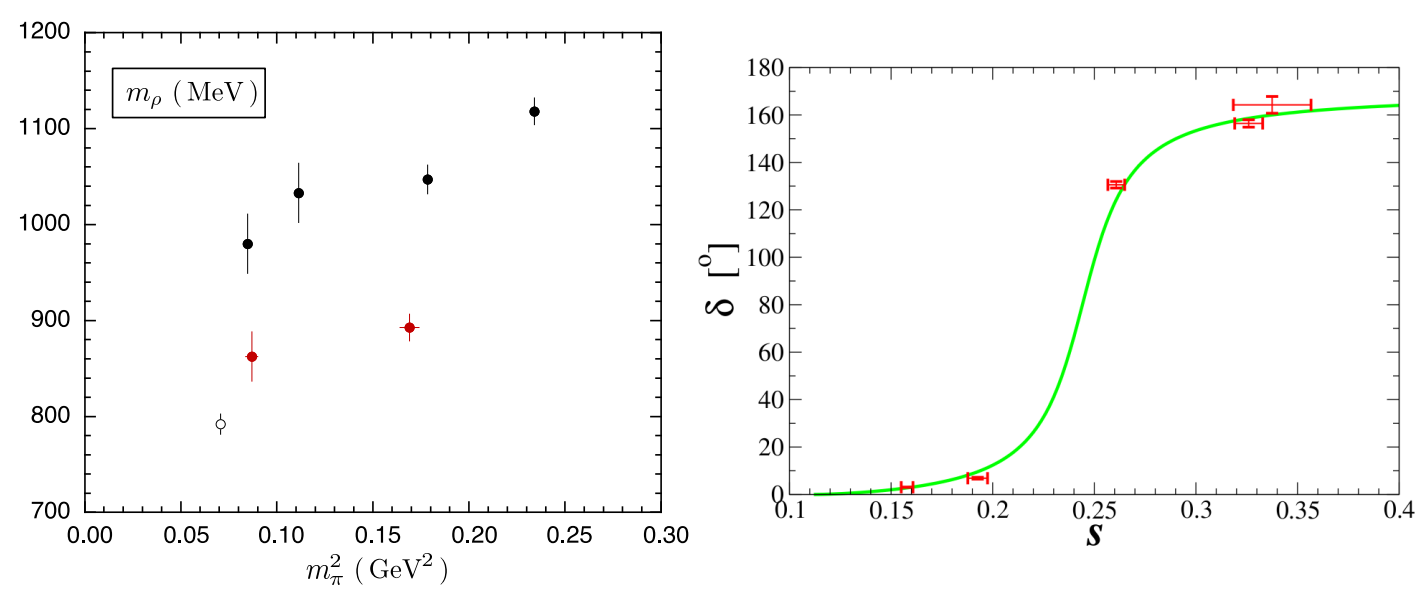

Figure 11: Results on the $\rho$ meson from unquenched ensembles. Left: A compilation of recent unquenched results taken from Ref. [33] (color online). The black points are taken from Ref. [34], the red points from Ref. [33], and the open circle from Ref. [35]. Right: The $I=1 \pi-\pi$ scattering phase shift using distillation (taken from Ref. [35]) on an $n_{f}=2$ ensemble with $a_{s}=a_{t}=0.12 \mathrm{fm}, m_{\pi}=260 \mathrm{MeV}$, and $L_{s}=1.9 \mathrm{fm}$. 

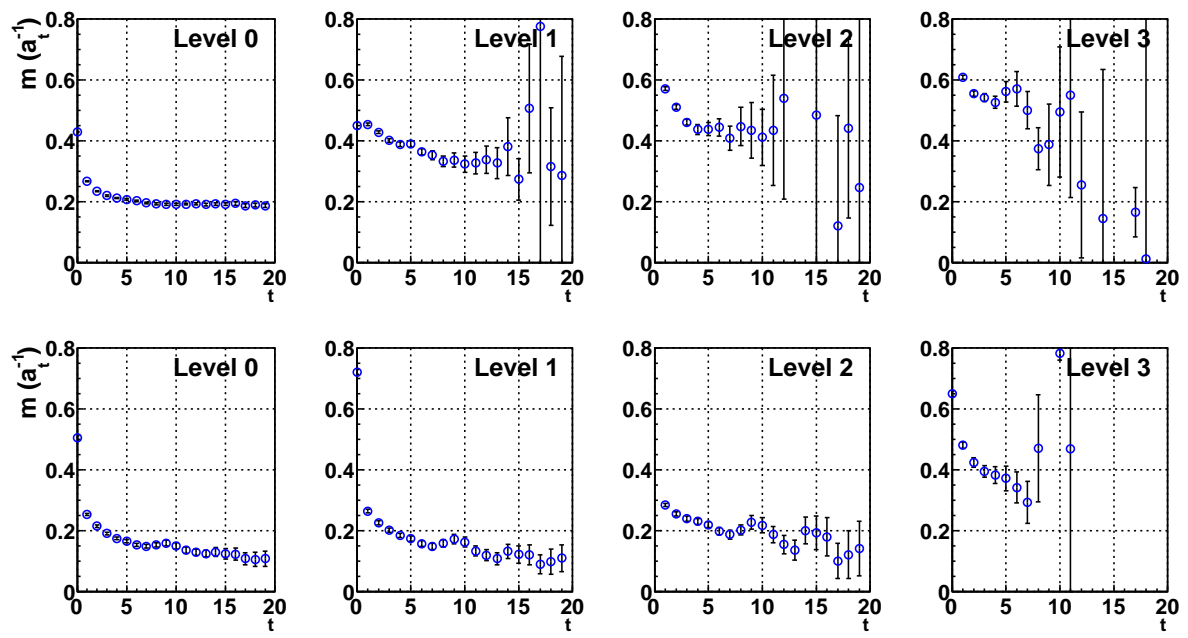

Figure 12: Preliminary results which require valence quark line disconnected diagrams using the stochastic LapH method. Top Row: Effective energies from a 5x5 GEVP in the Isoscalar pseudoscalar sector $(\eta$ mesons) with one unit of lattice momentum, taken from Ref. [38]. Results are from an $n_{f}=2+1$ ensemble with $a_{s}=3.5 a_{t}=0.12 \mathrm{fm}, m_{\pi}=400 \mathrm{MeV}$, and $L_{s}=1.9 \mathrm{fm}$. Bottom Row: Effective energies from a $4 \mathrm{x} 4$ GEVP in the isoscalar scalar sector (taken from Ref. [32]) on the same ensemble. The basis of operators used in this analysis consists of a local $\sigma$-meson operator, two $I=0 \pi-\pi$ meson operators, and a scalar glueball operator constructed from the low-lying eigenvalues of the gauge-covariant Laplace operator. The mixing between all three types of operators is significant.

preliminary results for moving $\eta$ mesons are shown in Fig. 12. Such a calculation is complicated by the reduced symmetry group of particles in motion and operators which transform according to irreps of the lattice little group must be constructed for various lattice momenta [37].

Fig. 12 also shows a first glimpse at a more realistic spectrum calculation in finite volume, specifically the isoscalar-scalar (vacuum) sector. This channel is particularly difficult as glueball states are present, in addition to single and multi-hadron states. A realistic calculation of the spectrum in this channel therefore requires interpolating fields with a reasonable overlap to these three types of states. Results of a preliminary such calculation are shown in Fig. 12. There a GEVP analysis was performed which contained two local ( $\sigma$-meson) operators, two $I=0 \pi-\pi$ operators with equal and opposite momenta, and a scalar glueball operator created using the low-lying eigenvalues of the gauge covariant Laplace operator. Indeed, significant mixing was found between all three types of operators in this channel.

Apart from finite volume energies, matrix elements of local operators between finite volume Hamiltonian eigenstates can also be calculated. In general, it is non-trivial to rigorously relate these quantities to those which have a well-defined infinite volume limit, but nonetheless these matrix elements may have phenomenological implications. Apart from the work presented here, preliminary calculations of transitions between excited and ground states (which neglect the effect of disconnected diagrams) have been performed in the nucleon and charmonium sectors [39, 40].

The situation is somewhat simplified for matrix elements of the light-light axial current between $B$ and $B^{*}$ mesons. First, no disconnected diagrams contribute to the required three-point correlation functions. Secondly, radial excitations of pseudoscalar static light mesons can only de- 

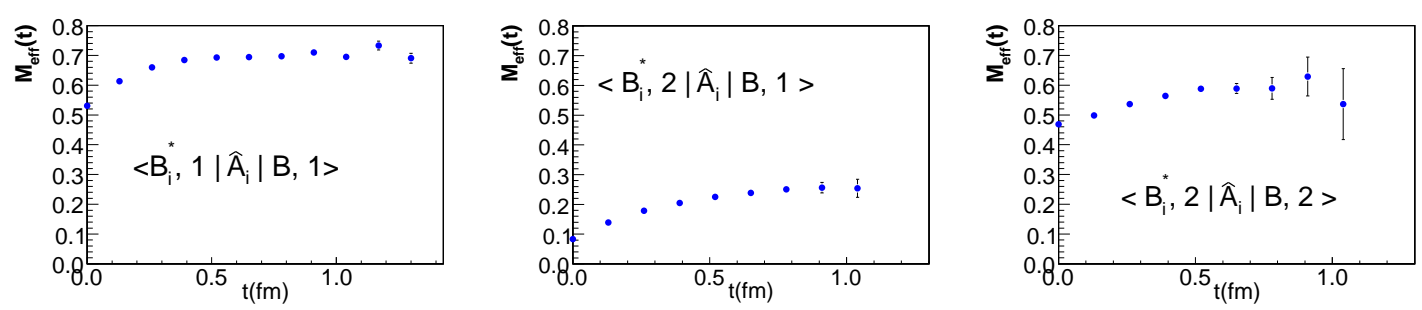

Figure 13: Effective matrix elements of the light-light axial current between radial excitations of $B^{*}$ and $B$ mesons in the static limit. These results (taken from Ref. [43]) are from an $n_{f}=2$ ensemble with $a_{s}=a_{t}=0.065 \mathrm{fm}, m_{\pi}=447 \mathrm{MeV}$, and $L_{s}=2.1 \mathrm{fm}$. Although the renormalization factors for this lattice discretization are known non-perturbatively, only bare matrix elements are shown here for illustrative purposes. Nonetheless, since the axial current is taken here at zero momentum transfer, these matrix elements are multiplicatively renormalized only and ratios between bare matrix elements are renormalized quantities.

cay strongly via the emission of two pions. At pion masses which are large enough so that the first radial excitation is significantly below the two-pion threshold, this excitation is stable and matrix elements of the axial current involving this state and the ground state have a well defined infinitevolume limit. Results from a preliminary calculation of these matrix elements are shown in Fig. 13 where rather than effective energies, effective matrix elements are plotted. These effective matrix elements $[15,16]$ are also obtained from solutions of the GEVP and are defined as

$$
M_{m n}^{e f f}(t)=R_{m}\left(t, t_{0}\right) R_{n}\left(t, t_{0}\right) \times\left(v_{m}\left(t, t_{0}\right), C^{3 p t}\left(t, t_{0}\right) v_{n}\left(t, t_{0}\right)\right)
$$

where parentheses denote an inner product over the GEVP indicies, $v_{m}\left(t, t_{0}\right)$ are GEVP eigenvectors, and $R_{m}\left(t, t_{0}\right)$ are normalization factors constructed to cancel the asymptotic time dependence. Unlike the effective energies, here the condition $t_{0}>t / 2$ is not required to reduce the asymptotic corrections, but $t$ must be at least larger than $t_{0}$. The asymptotic behavior of this effective matrix element is proven [15] to be $M_{m n}^{e f f}\left(t, t_{0}\right)=\left\langle B_{i}^{*}, m\left|\hat{A}_{i}\right| B, n\right\rangle+\mathscr{O}\left(\mathrm{e}^{-\left(E_{N+1}-E_{m, n}\right) t_{0}}\right)$, where $\hat{A}_{i}$ is the lightlight axial current, while $\left|B_{i}^{*}, m\right\rangle$ and $|B, n\rangle$ are finite volume Hamiltonian eigenstates corresponding to radial excitations of static-light zero-momentum $B^{*}$ and $B$ mesons, respectively.

The required matrix of three point correlation functions is given as $C_{i j}^{3 p t}(t)=\left\langle\mathscr{O}_{i}^{k}(2 t) A_{k}(t) \overline{\mathscr{O}}(0)\right\rangle$, where $\left\{\mathscr{O}_{i}^{k}\right\}$ and $\left\{\mathscr{O}_{i}\right\}$ are sets of interpolating operators for $B^{*}$ and $B$ mesons, respectively. Since the three-point correlation function contains two separations which both must be taken large, the condition $t_{0}<t$ means that in terms of the total separation $t_{s}=2 t$ the asymptotic correction to $M_{m n}^{e f f}\left(t, t_{0}\right)$ is $\mathscr{O}\left(\mathrm{e}^{-\left(E_{N+1}-E_{m, n}\right) t_{s} / 2}\right)$, compared with the $\mathscr{O}\left(\mathrm{e}^{-\left(E_{N+1}-E_{n}\right) t_{s}}\right)$ discussed earlier for effective energies.

It should be noted that for matrix elements for which $E_{m}=E_{n}$, the asymptotic corrections may be improved [41, 16, 42]. This entails the use of a summed insertion where instead of $C^{3 p t}\left(t_{1}, t_{2}\right)$, we employ $D^{3 p t}(t)=\sum_{t_{1}} C^{3 p t}\left(t+t_{1}, t_{1}\right)$. Using this summed insertion with the GEVP it is possible to obtain an asymptotic correction which is proven [16] to be $\mathscr{O}\left(\mathrm{e}^{-\left(E_{N+1}-E_{n}\right) t_{s}}\right)$, as in the effective energies. However, effective matrix elements constructed from this summed insertion are typically noisier, as a temporal derivate must be taken numerically.

In conclusion, excited hadron spectroscopy in Lattice QCD is a field which is currently evolving. While the systematic extraction of infinite volume resonance parameters from finite volume 
energy spectra remains a difficult problem, progress has been made in the extraction of finite volume energy spectra from lattice data. One of the major sources of difficultly in calculating finite volume energy spectra in Lattice QCD is the need to include both single and multi-hadron interpolating fields in a correlation matrix analysis. The requires the evaluation of valence quark line disconnected diagrams which require the knowledge of quark from all initial space-time points to all final space-time points.

Although all-to-all propagators cannot be calculated naively, an efficient stochastic algorithm (stochastic LapH) has been developed which requires a computational cost that scales linearly with the spatial volume. This technique (as well as others) has enabled preliminary calculations of spectra in systems which require disconnected diagrams, such as the $I=0$ scalar sector and the $\rho$-meson sector, where multi-hadron diagrams should be included in the basis of interpolating fields. Finally, apart from finite volume energies, transitions between finite volume Hamiltonian eigenstates can be calculated.

Acknowledgements. I gratefully thank the local and international organizing committees of Lattice 2011 for inviting me to talk and for providing a stimulating conference experience. Also, I am indebted to Rainer Sommer and K. Jimmy Juge for critical comments on the manuscript.

\section{References}

[1] Particle Data Group, K. Nakamura et al., J.Phys.G G37 (2010) 075021.

[2] C. Meyer and Y. Van Haarlem, Phys.Rev. C82 (2010) 025208, 1004.5516.

[3] SESAM Collaboration, G.S. Bali, H. Neff, T. Duessel, T. Lippert and K. Schilling, Phys.Rev. D71 (2005) 114513, hep-lat/0505012.

[4] M. Luscher and U. Wolff, Nucl.Phys. B339 (1990) 222.

[5] P. Giudice, D. McManus and M. Peardon, PoS LATTICE2010 (2010) 105, 1009.6192.

[6] HAL QCD Collaboration, S. Aoki et al., (2011), 1106.2281.

[7] V. Bernard, M. Lage, U.G. Meissner and A. Rusetsky, JHEP 0808 (2008) 024, 0806.4495.

[8] J.M. Bulava et al., Phys.Rev. D79 (2009) 034505, 0901.0027.

[9] J. Bulava et al., Phys.Rev. D82 (2010) 014507, 1004.5072.

[10] G. Bali et al., (2011), 1108.6147.

[11] G. Engel, These Proceedings .

[12] M.S. Mahbub, W. Kamleh, D.B. Leinweber, M.P. J. and A.G. Williams, to appear , ADP-11-37/T759.

[13] B.J. Menadue, W. Kamleh, D.B. Leinweber and M. Mahbub, (2011), 1109.6716.

[14] C. Michael and I. Teasdale, Nucl.Phys. B215 (1983) 433.

[15] B. Blossier, M. Della Morte, G. von Hippel, T. Mendes and R. Sommer, JHEP 0904 (2009) 094, 0902.1265.

[16] J. Bulava, M. Donnellan and R. Sommer, (2011), 1108.3774.

[17] S. Basak et al., Phys.Rev. D72 (2005) 094506, hep-lat/0506029. 
[18] Lattice Hadron Physics Collaboration (LHPC), S. Basak et al., Phys.Rev. D72 (2005) 074501, hep-lat/0508018.

[19] J.J. Dudek, R.G. Edwards, N. Mathur and D.G. Richards, Phys.Rev. D77 (2008) 034501, 0707.4162.

[20] M. Della Morte and L. Giusti, Comput.Phys.Commun. 180 (2009) 819, 0806.2601.

[21] M.G. Endres, D.B. Kaplan, J.W. Lee and A.N. Nicholson, (2011), 1106.0073, * Temporary entry *.

[22] S. Prelovsek et al., Phys.Rev. D82 (2010) 094507, 1005.0948.

[23] Hadron Spectrum Collaboration, M. Peardon et al., Phys.Rev. D80 (2009) 054506, 0905.2160.

[24] C. Morningstar et al., Phys.Rev. D83 (2011) 114505, 1104.3870.

[25] For the Hadron Spectrum Collaboration, J. Bulava et al., PoS LATTICE2010 (2010) 110, 1011.5277.

[26] J.J. Dudek, R.G. Edwards, M.J. Peardon, D.G. Richards and C.E. Thomas, Phys.Rev. D83 (2011) 071504, 1011.6352 .

[27] J.J. Dudek, R.G. Edwards, M.J. Peardon, D.G. Richards and C.E. Thomas, Phys.Rev. D82 (2010) 034508, 1004.4930 .

[28] J.J. Dudek et al., Phys.Rev. D83 (2011) 111502, 1102.4299.

[29] S. Ryan, These Proceedings .

[30] J. Foley et al., Comput.Phys.Commun. 172 (2005) 145, hep-lat/0505023.

[31] J. Foley et al., (2010), 1011.0481.

[32] J. Bulava et al., (2011), 1111.0845, * Temporary entry *.

[33] CS Collaboration, T.P..S. Aoki et al., (2011), 1106.5365, * Temporary entry *.

[34] X. Feng, K. Jansen and D.B. Renner, Phys.Rev. D83 (2011) 094505, 1011.5288.

[35] C. Lang, D. Mohler, S. Prelovsek and M. Vidmar, Phys.Rev. D84 (2011) 054503, 1105.5636.

[36] C. Pelissier, A. Alexandru and F.X. Lee, (2011), 1111.2314, * Temporary entry *.

[37] J. Foley, These Proceedings .

[38] D. Lenkner, These Proceedings .

[39] H.W. Lin, S.D. Cohen, R.G. Edwards and D.G. Richards, Phys.Rev. D78 (2008) 114508, 0803.3020.

[40] J.J. Dudek, R. Edwards and C.E. Thomas, Phys.Rev. D79 (2009) 094504, 0902.2241.

[41] S. Capitani, B. Knippschild, M. Della Morte and H. Wittig, PoS LATTICE2010 (2010) 147, 1011.1358.

[42] ALPHA Collaboration, J. Bulava, M. Donnellan and R. Sommer, PoS LATTICE2010 (2010) 303, 1011.4393.

[43] J. Bulava, M. Donnellan and B. Blossier, In preparation . 